\title{
Duty Cycle for Low Energy Operation of a Personal Conditioning Device
}

\author{
Rohit Dhumane Jiazhen Ling Vikrant Aute Reinhard Radermacher \\ Center for Environmental Energy Engineering, University of Maryland, College Park, 4164 Glenn L. Martin Hall \\ Bldg., MD 20742, USA \\ \{dhumane, jiazhen, vikrant, raderm\} @umd. edu
}

\begin{abstract}
The Roving Comforter (RoCo) is an innovative personal thermal management technology that provides ultimate personal thermal comfort for individuals in inadequately or even unconditioned environments. It is a miniature heat pump system mounted on a robotic platform capable of autonomously following individuals to deliver comfort by directing hot or cold air through automatically controlled nozzles. This allows buildings to relax their thermostats up to $4^{\circ} \mathrm{F}\left(2.2^{\circ} \mathrm{C}\right)$, leading to energy savings anywhere between 10 to $30 \%$ depending on climatic conditions. Since RoCo is a portable device, it needs to be operated on battery. A smaller battery pack will require frequent charging making it inconvenient for the users, while a bigger battery pack will add to the weight of the device leading to higher power consumption during motion. To address this problem, a multi-physics model for the operation that incorporates thermodynamics, electricity and mechanics of RoCo is developed and two duty cycles analyzed. Strategies for the operation of RoCo are provided from the observations of results.
\end{abstract}

Keywords: Battery, Air-conditioner, Duty-cycle

\section{Introduction}

Climate change and global warming have been hot topics of discussion over past few decades. The greenhouse gas emission from human activities has lead to disruption of several natural systems leading to rising sea-levels, increased ground instability in mountains and change in seasonal winds. The United Nations IPCC has identified the building industry as the one with the most climate mitigation potential (Intergovernmental Panel on Climate Change Fourth Assessment, 2007).

Building Heating, Ventilation and Air Conditioning (HVAC) account for $13 \%$ of energy consumption in the United States (United States Department of Energy, 2011). Much of this energy goes into maintaining narrow indoor temperature ranges that building operators consider necessary for comfort but are really not necessary for occupant comfort (Zhang et al., 2011).

Hoyt et al. (2015) demonstrated the potential of energy savings from extending thermostat set-points in the building. They concluded that if it were possible to relax the

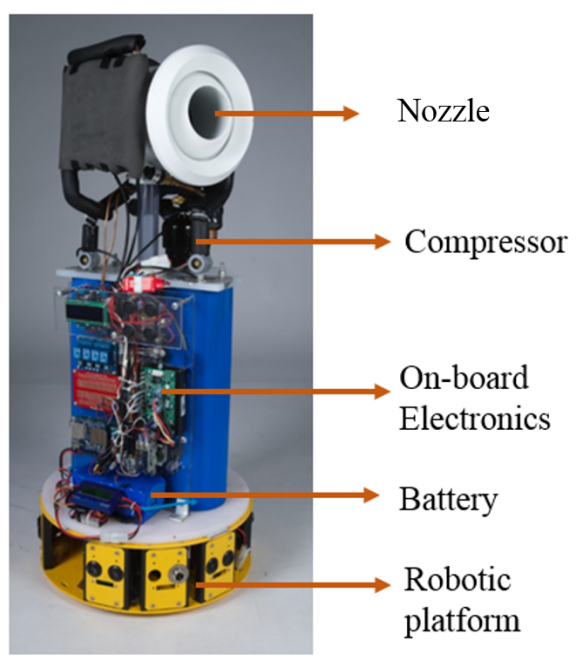

Figure 1. Current prototype of RoCo

temperature range in either the hot or cold direction, total HVAC energy is reduced at a rate of $10 \%$ per ${ }^{\circ} \mathrm{C}$. To enable expansion of building set-point temperatures, it is necessary to provide supplementary Personal Conditioning System (PCS) operating at significantly lower energy consumption.

PCS offer dual benefits of energy saving and increased comfort. As a result, several PCS have been developed and are summarized in the review articles by Zhang et al. (2015) and Veselý and Zeiler (2014). However, except for the desk and ceiling fans, they are not commercially available. This can be attributed to a variety of factors unique to the designs like poor thermal performance, low energy efficiency, high cost and poor aesthetics. To address these issues and to achieve the benefits of PCS, an innovative robotic personal conditioning device called the Roving Comforter (RoCo) shown in Figure 1, is being developed (Du et al., 2016).

\section{System Description}

In technical terms, RoCo is a vapor compression system mounted on an autonomous robotic platform and delivering comfort by directing hot or cold air using its automat- 


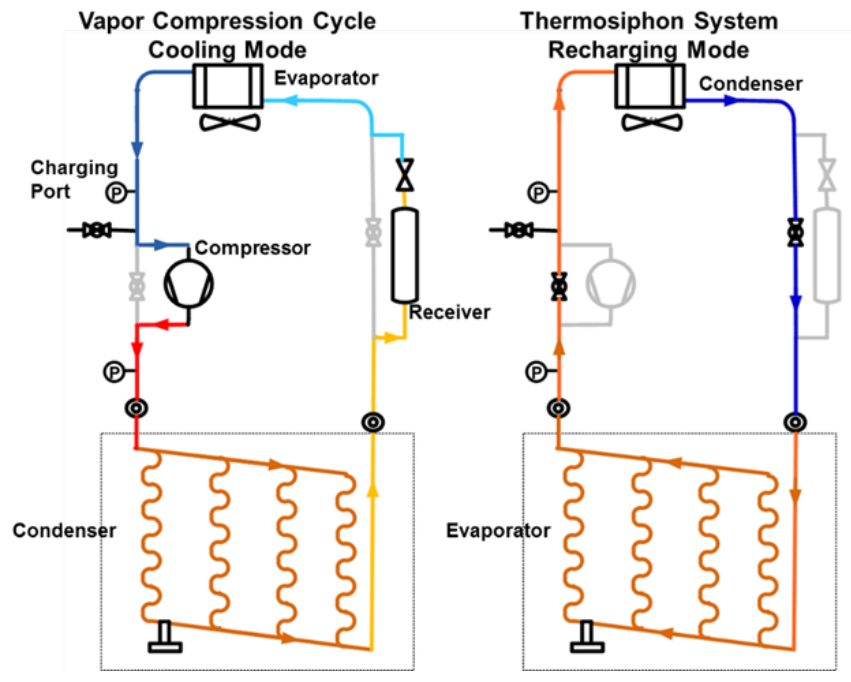

Figure 2. Conceptual representation of RoCo in operation

ically controlled nozzles. It is a heat pump on wheels.

RoCo stores its condenser heat in a compact phase change material based thermal storage which needs to be recharged before its next cooling operation. Thus RoCo operates in two modes and it is necessary to size the battery to deliver multiple operating cycles. Figure 2 shows the schematic of the two alternating modes of RoCo. The left schematic in Figure 2 shows the onboard vapor compression system using R134a as the refrigerant. The cooling operation is terminated when the PCM surrounding the condenser is completely melted. Before the next cooling operation, there is a need to re-solidify the PCM. This PCM recharge is achieved by a gravity assisted thermosiphon operation. Details of this recharge operation and its modeling are discussed in Dhumane et al. (2016).

The current prototype has separate battery packs for its robotic platform and vapor compression system. For the new prototype, a single unified battery pack is desired. An innovative nozzle design which permits nozzle rotation using motors in both horizontal and vertical plane is being developed for the new prototype. To understand various power draws for a single unified battery pack a duty cycle, representative of a worst-case operation of the new RoCo prototypes incorporating all the modifications is conceptualized. Simulations are carried out for the operation of various components to come up with strategies for increased the battery operation time.

\section{Component Modeling}

The model for the current simulation is shown in Figure 3. The top portion of the model contains components for modeling the vapor compression cycle. The compressor pumps the refrigerant in the circuit. A non-adiabatic tube element accounts for heat losses from the refrigerant between the compressor and the condenser. The condenser consists of inlet and outlet headers, and four refrigerant tubes immersed in PCM (See Figure 2 to understand the tube layout). The headers are modeled by lumped refriger- ant control volumes. The four refrigerant circuits formed by each of the refrigerant tube immersed in the PCM are assumed to be symmetric. To avoid computational expense, only one of these refrigerant circuits is modeled. Flow splitter and flow mixer components are used to accomplish this. The splitter component divides the refrigerant mass flow rate into four equal parts and the mixer merges it back to resume the original mass flow rate for the next component in the refrigerant circuit. The PCM blocks interact with the refrigerant control volume using the HeatPort interface. The refrigerant then flows through receiver, valve and evaporator before reaching the compressor. Component models for refrigerant tube connecting various components are also included.

The bottom left portion contains the battery model and a power load component. The latter needs power draw as an input. The components which draw current from the battery are Compressor, fan, nozzle, robotic platform and on-board electronics. The power consumption from all of them is added and provided as input for the power load component. It then determines the current draw from the battery. This section discusses equations involved in calculating the power consumption from all these components.

\subsection{Vapor Compression System Components}

RoCo is a heat pump on wheels and detailed modeling for vapor compression cycle is carried out using components from CEEE Modelica Library (CML) (Qiao et al., 2015). Components used from the library are compressor, evaporator, pipe, receiver and fixed orifice expansion device.

\subsection{Phase Change Material Heat Exchanger}

The condenser consists of helical refrigerant tubes surrounded by the phase change material (PCM). The PCM melting is a complex phenomenon due to the fact that the solid-liquid boundary moves depending on the rate of heat transfer and hence its position with time forms part of the solution. The rate of heat transfer varies progressively during the melting due to varying effects of conduction and natural convection. It decreases in at early times, attains a minimum, then rises again to a maximum and subsequently decreases (Sparrow et al., 1977). The helical nature of the refrigerant tube further increases the complexity by making the problem 3D. Due to strong non-linear nature of the problem, a simple $2 \mathrm{D}$ problem of melting in a square cavity may take several days on a personal computer (Wang et al., 2010). Finally, the two-phase refrigerant circuit exchanging heat with the PCM adds difficulty in convergence.

The model used in the current work is a trade-off for accuracy, complexity and usability. The PCM block is taken as a lumped control volume to eliminate the momentum equation. Two components are used to model PCM: PCMConductor and PCMCapacitor which are PCM analogous versions of HeatCapacitor and Thermalconductor from the Thermal package of Modelica Standard Library. Temperature transforming model by Cao and Faghri (1990) is used to model the energy equation since it also captures temperature glide over melting without much oscillatory effects. A heat transfer coefficient vs melt fraction profile based on the various heat transfer regimes discussed in Sparrow et al. (1977) is used and fitted to match experimental data. 


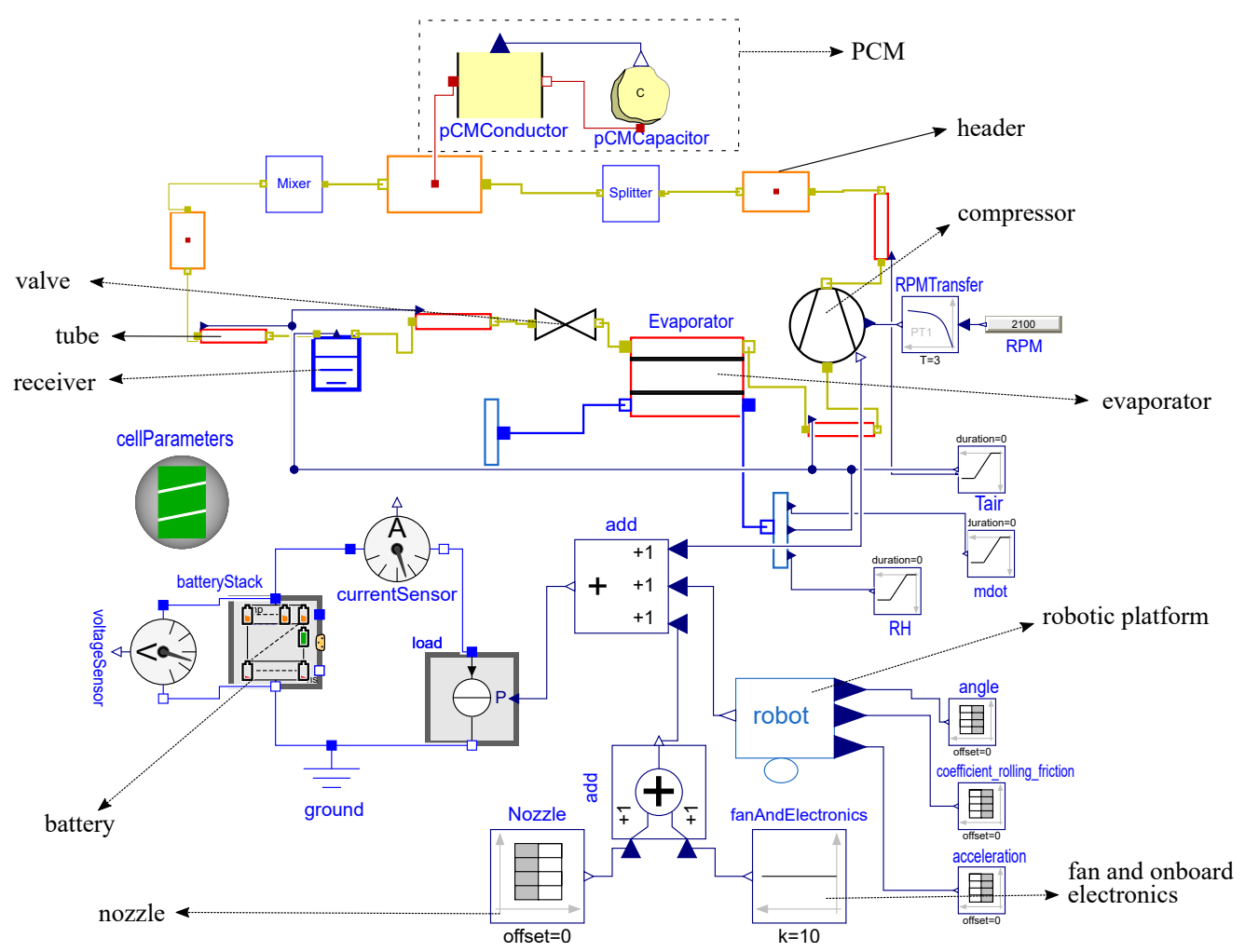

Figure 3. Schematic of System Model for RoCo.

\subsubsection{PCM Capacitor}

The PCM Capacitor block includes a HeatPort and models the heat storage of PCM. The equations for temperature transforming model use scaled temperature $\left(T^{*}[\mathrm{~K}]\right)$ as input. It is defined as:

$$
T^{*}=T-T_{m}
$$

where $T_{m}[\mathrm{~K}]$ is the mid-point of the temperature glide and $T$ $[\mathrm{K}]$ is the lumped PCM temperature. The specific enthalpy ( $h$ $\left.\left[\mathrm{J} \mathrm{kg}^{-1}\right]\right)$ is calculated by:

$$
h=c\left(T^{*}+s\right)
$$

The specific heat capacity $\left(c\left[\mathrm{~J} \mathrm{~kg}^{-1} \mathrm{~K}^{-1}\right]\right)$ and the source term $(s[\mathrm{~K}])$ are defined as:

$$
c= \begin{cases}c_{s}, & T^{*}<-\delta T \\ \frac{c_{s}+c_{l}}{2}+\frac{h_{s l} l}{2 \delta T}, & -\delta T \leq T^{*} \leq \delta T \\ c_{l}, & T^{*}>\delta T\end{cases}
$$

where, $\delta T[\mathrm{~K}]$ is the temperature range over which the PCM melts.

$$
s= \begin{cases}\delta T, & \text { if } T^{*} \leq \delta T \\ \frac{c_{s}}{c_{l}} \delta T+\frac{h_{s l}}{c_{l}}, & T^{*}>\delta T\end{cases}
$$

The if-else loops are implemented using NoEvent operator as shown below for the specific heat capacity block.

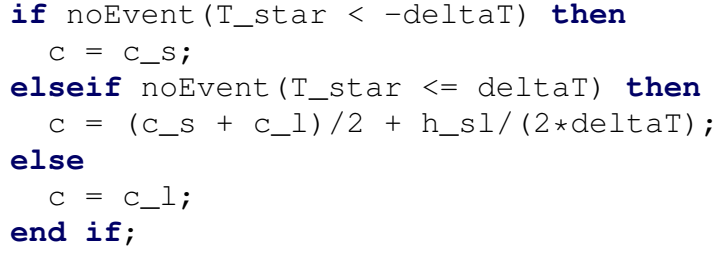

The melt fraction $(\lambda)$ of PCM is calculated from its enthalpy value as:

$$
\lambda=\max \left(0, \min \left(1, \frac{h}{h_{l}}\right)\right.
$$

where $h_{l}\left[\mathrm{~J} \mathrm{~kg}^{-1}\right]$ is the enthalpy at the point where the PCM just turns liquid. The equation is simplified because of the fact that the enthalpy scale is defined as zero for the point where the PCM starts to melt. The melt fraction is made available for the PCM capacitor block through the Realoutput interface.

\subsubsection{PCM Conductor}

PCM Conductor block connects the refrigerant control volume of the condenser to the PCM Capacitor block. It extends Modelica. Thermal. HeatTransfer. Interfaces. Element1D block and provides for the heat flow, which is calculated using CombiTable1D fitted function for heat transfer coefficient as a function of melt fraction. The Realinput interface is used to obtain melt fraction input from PCM Capacitor.

Table 1 contains the anchor points given to the CombiTable block used as input for the normalized heat transfer coefficient 
as a function of melt fraction. The constant value used to multiply the normalized function to obtain heat transfer coefficient (HTC) is $116 \mathrm{~W} \mathrm{~m}^{2} \mathrm{~K}^{-1}$. These numbers are obtained by matching the condenser pressure from simulation to the experiment since there are no correlations to capture the behavior in literature. Pal and Joshi (2001) discusses the heat transfer variation in the four regimes captured by Table 1 . The initial heat transfer occurs in a conduction dominated regime. Then there is a reduction in heat transfer coefficient with the appearance of small melt layer because the velocity of the liquid PCM due to buoyancy force is low. The melting then progresses to a convection dominated regime where the velocity of liquid PCM increases causing a higher rate of heat transfer. Finally, the magnitude of velocity decreases as the temperature in the molten PCM becomes more uniform with time due to natural convection stirring, leading to reduced buoyancy force for convection.

Table 1. Input table for PCM Conductor block.

\begin{tabular}{cc}
\hline Melt Fraction & Normalized HTC \\
\hline 0 & 1 \\
0.2 & 0.9 \\
0.4 & 1 \\
0.7 & 0.9 \\
1 & 0.8 \\
\hline
\end{tabular}

\subsection{Battery}

Battery modeling is necessary to reduce the total weight and cost, which are critical parameters in the design of RoCo. Accurate prediction of the state of charge (SOC) of the battery is essential to determine how long the battery will last with a typical user case scenario. The battery capacity should be sufficient to run the whole cooling operation with charging and discharging operations without entering regions of overcharging and overdischarging for longer operation.

There are a variety of methods for mathematical modeling of the battery which vary in complexity, computational requirements and reliability of the prediction. The models based on electrochemical principles which model first-principle phenomena require significant computational resources and detailed datasets for input (Marco et al., 2015). Equivalent circuit models have a good trade-off between exactness, complexity and usability while still providing some insights into the battery state (Einhorn et al., 2011b). As a result, the equivalent circuit approach is used for the current research. Modeling for the battery is carried out using the Electrical Energy Storage Library (Einhorn et al., 2011a). The battery pack is modeled using the model LinearDynamicImpedance from the battery stack sub-package.

The battery model in the library involves modeling a single cell as an effective resistance capacitor [R-C] circuit. By taking inputs of the number of cells in series and number of cells in parallel of the battery, the behavior of the battery can be modeled by appropriate scaling of the cell model.

The state of charge of a cell (SOC) is calculated as:

$$
S O C=S O C_{0}-\frac{\Delta Q}{C}
$$

where, $\Delta Q[\mathrm{C}]$ is the charge removed and $C[\mathrm{C}]$ is the capacity of the cell. $S O C_{0}[\mathrm{C}]$ is the initial state of charge of the cell.

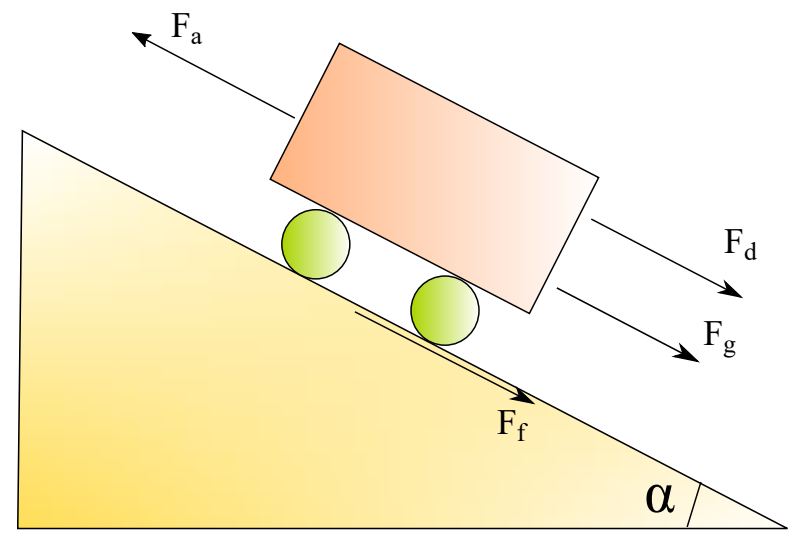

Figure 4. Free Body Diagram for Robotic platform

The model provides inputs for modeling the capacity of the cell, resistances and capacitances as variables using parameters for calendaric aging and aging due to cycling. The charge removed from the battery is calculated as shown in Equation (7).

$$
\Delta Q=\int_{0}^{t} I d t
$$

Various components are available to model different types of loads in the circuit, which determines the current $I$ [A]. For the present study, the component SignalPower is used. This component takes power draw as input and creates loads on the battery accordingly.

\subsection{Platform}

The power consumption from the motion of Robotic platform is estimated from first-principles based approach used by (Gonullu, 2013). The free body diagram of the platform is shown in Figure 4 . The total force required for motion $\left(F_{t}[\mathrm{~N}]\right)$ is the sum of gravitational force acting along the incline $\left(F_{g}[\mathrm{~N}]\right)$, the drag force by the air $\left(F_{d}[\mathrm{~N}]\right)$, the force to overcome friction $\left(F_{f}[\mathrm{~N}]\right)$ and the force required to produce acceleration $\left(F_{a}[\mathrm{~N}]\right)$.

$$
F_{t}=F_{g}+F_{d}+F_{f}+F_{a}
$$

Since the platform operates at speeds of around $1 \mathrm{~ms}^{-1}$, the drag force $\left(F_{d}\right)$ can be neglected. If $m[\mathrm{~kg}]$ is the total mass of RoCo, $g\left[\mathrm{~m} \mathrm{~s}^{-2}\right]$ the acceleration due to gravity, the remaining

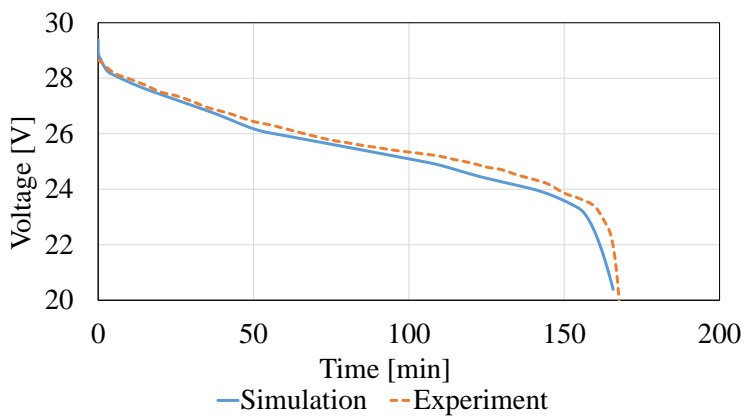

Figure 5. Comparison of simulation results to experimental for the battery discharge test 
forces can be calculated as:

$$
\begin{aligned}
& F_{g}=m g \sin (\alpha) \\
& F_{f}=f_{r} m g \cos (\alpha) \\
& F_{a}=m a
\end{aligned}
$$

Finally, the net power for the motion of the platform $(P[\mathrm{~W}])$ is obtained as:

$$
P=F_{t} v
$$

where $v\left[\mathrm{~m} \mathrm{~s}^{-1}\right]$ is the velocity of the platform.

Typical variations in the motion are changes in velocity, motion over different surfaces and on surfaces with different inclinations. A TimeTable block for each of acceleration $(a$ $\left.\left[\mathrm{ms}^{-2}\right]\right)$, rolling friction resistance of surface $\left(f_{r}\right)$ and inclination angle of surface with horizontal $(\alpha)$ is used to capture these variations in the motion.

\subsection{Fan}

The fan operates continuously during the operation of RoCo and can be modeled by Modelica.Blocks.Sources.Constant. The power measured from the experiment is given as input. The power draw from on-board electronics is also constant and is lumped together with the fan power.

\subsection{Nozzle}

The stationary nozzle modeled in Figure 1 can be housed with two motors to rotate it along horizontal and vertical directions. The power consumed by the rotary nozzle is taken as the maximum power draw of two DC motors used for its motion. The transient power draw from the nozzle is given by Modelica.Blocks. Sources. TimeTable block. The nozzle is assumed to move continuously for a period of $10 \mathrm{sec}-$ onds, for every 5-minute interval.

\section{System Model}

The screenshot for the system model is shown in Figure 3. The inputs for various components are discussed in this section. The displacement volume and RPM for the compressor are provided from manufacturer's data. The efficiency is adjusted to match the power consumption measured from experiment ( $\mathrm{Du}$ et al., 2016). For pressure drop calculations, values are calculated using various correlations (McAdams et al., 1942; Friedel, 1979; Lockhart and Martinelli, 1949; Müller-Steinhagen and Heck, 1986) and nominal values selected based on the range calculated from them. For refrigerant heat transfer coefficient, single phase heat transfer coefficients are evaluated using Dittus and Boelter (1985) correlation. Two phase heat transfer coefficient in the evaporator is evaluated using Shah (1982) correlation. Condenser consists of helical coils inside the PCM. The single phase liquid only heat transfer coefficient is calculated using Schmidt (1967) correlation to be used as input to Shah (2016) correlation for two phase heat transfer coefficient calculation. The air side heat transfer coefficients are calculated using Wang et al. (2000).

The battery used in the prototype shown in Figure 1 consists of 21 cells, consisting of 3 parallel lines of 7 cells of Samsung ICR 18650-26F in series (7s 3p). Its capacity is $7.8 \mathrm{Ah}$. Obtaining the input parameters for battery requires results from extensive battery testing carried out primarily to obtain parameters for the model which were not available in open literature. To

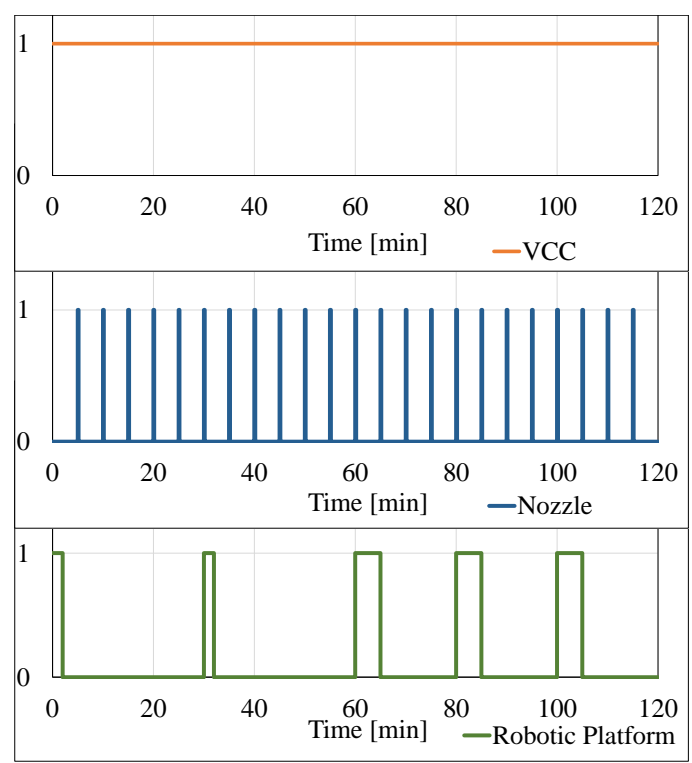

Figure 6. Typical operation of RoCo with VCC always ON

address this, the resistance and capacitance values for the model are taken from Muenzel et al. (2015) for Sanyo UR18650FM since it has similar cell capacity and initial internal impedance as Samsung ICR18650-26F. These two parameters are most important for modeling overall battery performance as can be seen from results of Einhorn et al. (2011b). For accurate prediction of battery performance, a battery discharge test is conducted using a constant power drawing circuit. The power draw of this circuit is selected to be similar to that of RoCo during a steady operation. The SOC vs OCV (Open Circuit Voltage) table is modified in the model to match the experimental discharge profile. The comparison of voltage discharge profile of simulation and experimental case is shown in Figure 5.

The weight of the system applied to the platform is taken to be $30 \mathrm{~kg}$. RoCo is assumed to be moving upwards a slope with $10^{\circ}$ incline. The coefficient of rolling friction is taken to be 0.05 which applies for poor condition stone paving to represent the worst conditions.

Two duty cycles are considered for the operation. The logic for the operation of various power draw sources in a two hour time duration is shown in Figure 6 and Figure 7. In the first one, the fan and compressor continue to operate for the whole duration (hereby referred to as Cycle 01, see Figure 6) while in the second one, they are stopped when RoCo is in motion (hereby referred to as Cycle 02, see Figure 7). This is done to avoid peaks in the power draws for battery. In the simulation, the compressor and fan are first turned off. The platform motion is started after a delay of 1 second.

\section{Results and Discussion}

The model is simulated using Dymola 2017 with Radau IIa order 5 stiff solver. The tolerance selected for the solver is $1 \mathrm{e}-6$. The simulation time on a PC with 16 GB RAM, 64-bit Operating System and $3.5 \mathrm{GHz}$ is 1159 seconds.

Figure 8 and Figure 9 show comparison of the simulated results with the experimental data. It can be observed that the simulation predicts the experimental trends to a reasonable extent. 


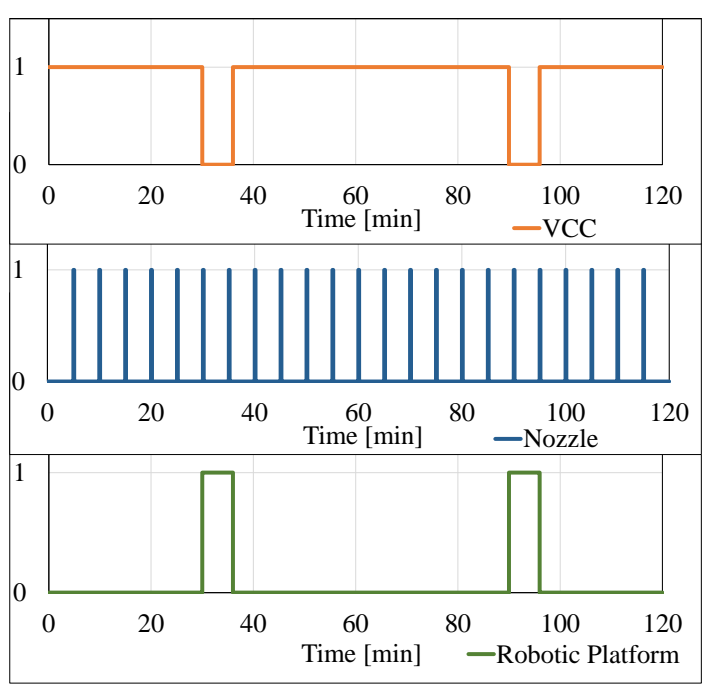

Figure 7. RoCo Operation with VCC turned off during motion

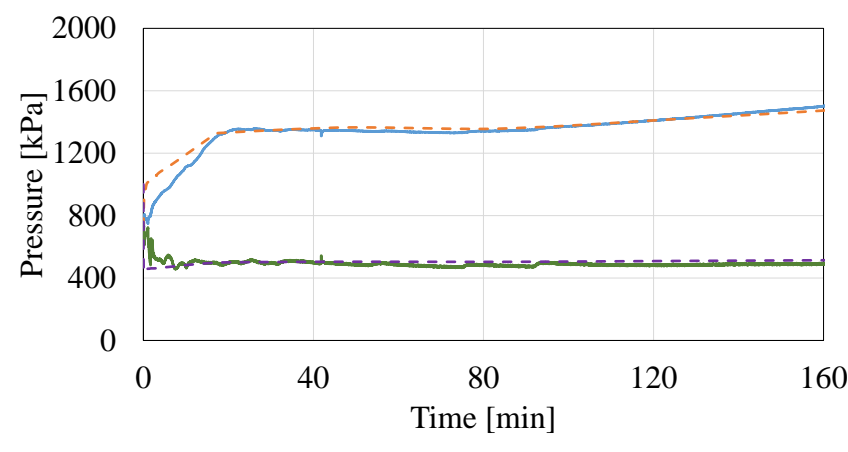

-Expt Discharge - -Sim Discharge - Expt Suction - -Sim Suction

Figure 8. Pressures at suction and discharge of compressor

The experimental setup uses a variable expansion valve which is modulated by an operator. The valve model used in the simulation assumes fixed opening and constant discharge coefficient. These two effects lead to the deviations in measured pressures and mass flow rates in the initial part of the operation. Another factor for the deviation of discharge pressure in the initial 20 minutes (Figure 8) is from the inaccuracy of the heat transfer coefficient.

The dynamic modeling for cycle 02 is complicated due to cycling. So to model it, the power consumption profile of compressor is extracted from cycle 01 results. For the portion where RoCo is in motion, the values are set to zero in the profile. This load is now given as an input to the battery using a Modelica.Blocks. Sources. TimeTable block.

The results of power consumption can be seen in Figure 10. It can be observed that in cases where all the components operate power exceeds $100 \mathrm{~W}$. However, by turning off the fan and compressor when RoCo is in motion, it is possible to limit the power draw to $70 \mathrm{~W}$.

For improved results, a few parameters from the simulation are calibrated for a better match with the experimental data ( $\mathrm{Du}$ et al., 2016). A fixed opening valve is used in the model. Its

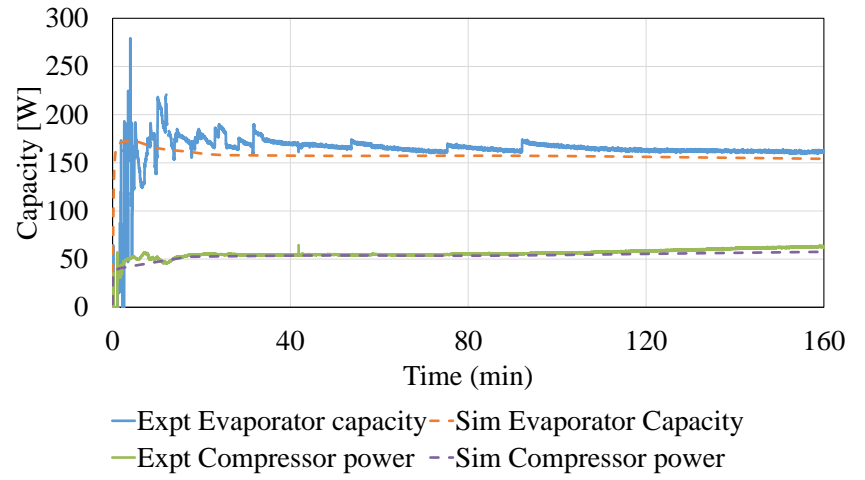

Figure 9. Cooling capacity and power consumption of RoCo

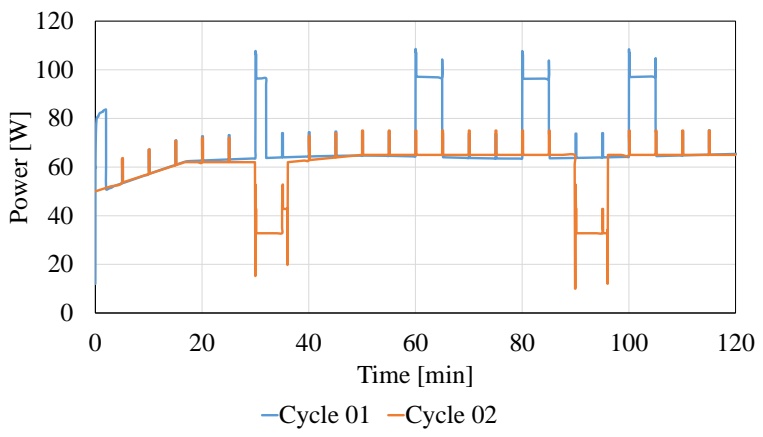

Figure 10. Power draw during the two operating cycles

opening and flow coefficient are adjusted using the experimental refrigerant mass flow rate. The suction and discharge pressure from simulation also need to be noted during the tXv parameter calibration. Since the RPM and displacement volume of compressor are available from manufacturer's data, the valve as the only component to significantly affect the refrigerant mass flow rate, permitting the aforementioned adjustment. Calibration is also needed for the battery, compressor power and PCM, which is already discussed in the respective sections.

\section{Conclusions}

A first principle based multi-physics model is developed to model the behavior of a portable air conditioning device. The model is used to capture power consumption of new version of the device for two different operating cycles. Based on the results of power consumption, it is observed that turning off the VCC during the motion can reduce peak load on the battery by up to $34 \%$.

\section{Acknowledgment}

This research was supported by the Advanced Research Projects Agency - Energy (ARPA-E) with Award Number DEAR0000530. We thank the members of Center for Environmental Energy Engineering (CEEE) and team members of the Roving Comforter Project for their support.

\section{References}

Y. Cao and A. Faghri. A numerical analysis of phase-change problems including natural convection. Journal of heat transfer, 112(3):812-816, 1990. doi:10.1115/1.2910466. 
R. Dhumane, Y. Du, A. Mallow, K. Gluesenkemp, J. Ling, V. Aute, and R. Radermacher. Transient Modeling of a Thermosiphon based Air Conditioner with Compact Thermal Storage: Modeling and Validation. In 16th International Refrigeration and Air Conditioning Conference, Purdue, Indiana, USA, 2016.

F. W. Dittus and L. M. K. Boelter. Heat transfer in automobile radiators of the tubular type. International Communications in Heat and Mass Transfer, 12(1):3-22, 1985. doi:10.1016/0735-1933(85)90003-X.

Y. Du, J. Muehlbauer, J. Ling, V. Aute, Y. Hwang, and R. Radermacher. Rechargeable Personal Air Conditioning Device. In ASME 2016 10th International Conference on Energy Sustainability collocated with the ASME 2016 Power Conference and the ASME 2016 14th International Conference on Fuel Cell Science, Engineering and Technology. American Society of Mechanical Engineers, 2016. doi:10.1115/ES2016-59253.

M. Einhorn, F. V. Conte, C. Kral, C. Niklas, H. Popp, and J. Fleig. A modelica library for simulation of electric energy storages. In Proceedings of the 8th International Modelica Conference; March 20th-22nd; Technical Univeristy; Dresden; Germany, number 63, pages 436-445. Linköping University Electronic Press, 2011a. ISBN 16503740. doi:10.3384/ecp11063436.

M. Einhorn, V. Conte, C. Kral, and J. Fleig. Comparison of electrical battery models using a numerically optimized parameterization method. In 2011 IEEE Vehicle Power and Propulsion Conference, pages 1-7. IEEE, 2011b. ISBN 1612842488. doi:10.1109/VPPC.2011.6043060.

L. Friedel. Improved friction pressure drop correlations for horizontal and vertical two-phase pipe flow. In European twophase flow group meeting, Paper E, volume 2, page 1979, 1979.

M. K. Gonullu. Development of a mobile robot to be used in mobile robot research. Master's thesis, Department of Mechanical Engineering, Middle East Technical University, 22013.

T. Hoyt, E. Arens, and H. Zhang. Extending air temperature setpoints: Simulated energy savings and design considerations for new and retrofit buildings. Building and Environment, 88: 89-96, 2015. doi:10.1016/j.buildenv.2014.09.010.

I. Intergovernmental Panel on Climate Change Fourth Assessment. Contribution of Working Groups I, II and III to the Fourth Assessment Report of the Intergovernmental Panel on Climate Change, 2007. ISSN 14764687. URL http: //www.ipcc.ch/publications \{_\} and__\}data/ ar4/syr/en/spms2.html \{\#\} footnote5.

R. W. Lockhart and R. C. Martinelli. Proposed correlation of data for isothermal two-phase, two-component flow in pipes. Chem. Eng. Prog, 45(1):39-48, 1949.

J. Marco, N. Kumari, W. D. Widanage, and P. Jones. A cellin-the-loop approach to systems modelling and simulation of energy storage systems. Energies, 8(8):8244-8262, 2015. doi:10.1049/cp.2011.0421.
W. H. McAdams, W. K. Woods, and L. C. Heroman. Vaporization inside horizontal tubes-II-benzene-oil mixtures. Trans. ASME, 64(3):193-200, 1942.

V. Muenzel, A. F. Hollenkamp, A. I. Bhatt, J. de Hoog, M. Brazil, D. A. Thomas, and I. Mareels. A Comparative Testing Study of Commercial 18650-Format LithiumIon Battery Cells. Journal of The Electrochemical Society, 162(8):A1592-A1600, 2015. ISSN 0013-4651. doi:10.1149/2.0721508jes.

H. Müller-Steinhagen and K. Heck. A simple friction pressure drop correlation for two-phase flow in pipes. Chemical Engineering and Processing: Process Intensification, 20 (6):297-308, 1986. ISSN 0255-2701. doi:10.1016/02552701(86)80008-3.

D. Pal and Y. K. Joshi. Melting in a side heated tall enclosure by a uniformly dissipating heat source. International Journal of Heat and Mass Transfer, 44(2):375-387, 2001. ISSN 00179310. doi:10.1016/S0017-9310(00)00116-2.

H. Qiao, V. Aute, and R. Radermacher. Transient modeling of a flash tank vapor injection heat pump system-part I: model development. International journal of refrigeration, 49:169182, 2015. doi:10.1016/j.ijrefrig.2014.06.019.

E. F. Schmidt. Wärmeübergang und Druckverlust in rohrschlangen. Chemie Ingenieur Technik, 39(13):781-789, 1967. doi:10.1002/cite.330391302.

M. M. Shah. Chart correlation for saturated boiling heat transfer: equations and further study. ASHRAE Trans.;(United States), 88(CONF-820112-), 1982.

M. M. Shah. Comprehensive correlations for heat transfer during condensation in conventional and mini/micro channels in all orientations. International journal of refrigeration, 67: 22-41, 2016. doi:10.1016/j.ijrefrig.2016.03.014.

E. M. Sparrow, S. V. Patankar, and S. Ramadhyani. Analysis of melting in the presence of natural convection in the melt region. Journal of Heat Transfer, 99(4):520-526, 1977. ISSN 0022-1481. doi:10.1115/1.3450736.

United States Department of Energy. Energy Efficiency and Renewable Energy. 2011.

M. Veselý and W. Zeiler. Personalized conditioning and its impact on thermal comfort and energy performance - A review. Renewable and Sustainable Energy Reviews, 34:401408, 2014. doi:10.1016/j.rser.2014.03.024.

C.-C. Wang, K.-Y. Chi, and C.-J. Chang. Heat transfer and friction characteristics of plain fin-and-tube heat exchangers, part II: Correlation. International Journal of heat and mass transfer, 43(15):2693-2700, 2000. doi:10.1016/s00179310(99)00333-6.

S. Wang, A. Faghri, and T. L. Bergman. A comprehensive numerical model for melting with natural convection. International Journal of heat and mass transfer, 53(9-10):19862000, 2010. doi:10.1016/j.ijheatmasstransfer.2009.12.057. 
H. Zhang, E. Arens, and W. Pasut. Air temperature thresholds for indoor comfort and perceived air quality. Building Research \& Information, 39(2):134-144, 2011. doi:10.1080/09613218.2011.552703.

H. Zhang, E. Arens, and Y. Zhai. A review of the corrective power of personal comfort systems in non-neutral ambient environments. Building and Environment, 91:15-41, 2015. doi:10.1016/j.buildenv.2015.03.013. 